Original Article

\title{
The effect of different imitation models on the accuracy and speed of imitation of movement
}

\author{
Hitomi Nishizawa, RPT, MS ${ }^{1)}$, Teij Kimura, RPT, PhD ${ }^{1)^{*}}$, Аh-Cheng Goh, $\mathrm{PhD}^{1)}$ \\ 1) School of Health Sciences, Faculty of Medicine, Shinshu University: 3-1-1 Nagano, Asahi, \\ Matsumoto 390-8621, Japan
}

\begin{abstract}
Purpose] The purpose of this study was to compare the accuracy, speed and subjective ease of imitation of movement using three different imitation models. [Subjects] Thirty-four right-handed healthy males participated in this study. [Methods] The imitation task chosen for this study was an asymmetric combined motion of the upper and lower limbs. Three kinds of imitation models were displayed on a screen as follows: a) third person perspective mirror imitation (3PM), b) third person perspective anatomical imitation (3PA), and c) first person perspective ipsilateral imitation (1PI). Subjects were instructed to imitate the movement shown on a screen as quickly and as accurately as possible. They executed four sets of the movement with each set consisting of one trial of each of the three imitation models. [Results] 3PM was the most accurate, and 1PI was the fastest in speed and subjective ease of imitation, compared with the other two imitation models. [Conclusion] These results suggest that 1PI and 3PM, which do not require mental rotation of the movement task as required by 3PA, should be considered more suitable imitation models for teaching healthy subjects how to move.

Key words: Movement imitation, Speed, Accuracy
\end{abstract}

(This article was submitted Jul. 8, 2015, and was accepted Aug. 5, 2015)

\section{INTRODUCTION}

In physical therapy clinical practice, instructions such as verbal commands or visual demonstrations are used to help patients learn or re-learn movement strategies and regain motor control ${ }^{1)}$. Among these different types of instructions, physical therapists (PT) use various types of imitation models to demonstrate the movement in order to get the patient to perform the same movement by imitating the PT. Imitation of movement is defined as the ability to observe and repeat the behavior of others, and is one of the principal modes of acquiring new patterns of behavior ${ }^{2}$. In previous studies of the demonstration and imitation of movement, it has been reported that the difference in the types of imitation models may affect the degree of imitation related to motor control and motor learning ${ }^{1,3-5)}$.

The results of these previous studies suggest that the accuracy of movement imitation may be affected by the manner in which the demonstration is performed by the PT. This may indicate the importance of the relationship between the type of imitation model used by the PT and the ability to imitate the movement by the patient ${ }^{4,6,7)}$.

There are several ways to classify imitation models used

*Corresponding author. Teiji Kimura (E-mail: tkimura@ shinshu-u.ac.jp)

(C2015 The Society of Physical Therapy Science. Published by IPEC Inc. This is an open-access article distributed under the terms of the Creative Commons Attribution Non-Commercial No Derivatives (by-ncnd) License $<$ http://creativecommons.org/licenses/by-nc-nd/3.0/>. by a PT. For example, imitation from the perspective of the participant ${ }^{8)}$, also known as first person perspective imitation (1PPI); or imitation as if watching from the perspective of someone else ${ }^{8}$, also known as third person perspective imitation (3PPI). Imitation models can also be classified as mirror imitation or anatomical imitation, according to the presence or absence of mental rotation. Shepard and Metzler $^{9)}$ described mental rotation as the act of someone viewing an object and rotating the object in his mind so that it is orientated in the same manner as the object being viewed. Franz et al. ${ }^{10)}$ used the term mirror imitation to refer to the performer mimicking the model as if the performer was looking into a mirror, and performing a right hand movement when the model moves her left hand, and a left hand movement when the model moves her right hand. In contrast, Koski et al. ${ }^{11)}$ used the term anatomical imitation to refer to a non-mirror mode of responding in which a participant imitates a model by moving the anatomically corresponding hand ${ }^{10)}$. Therefore, mirror imitation refers to imitation without using mental rotation and anatomical imitation refers to imitation using mental rotation.

Previous studies comparing 1PPI and 3PPI have reported that $1 \mathrm{PPI}$ is more accurate and faster than $3 \mathrm{PPI}^{8}$. On the other hand, in studies comparing mirror imitation and anatomical imitation, Franz et al. ${ }^{10)}$ and Chiavarino et al. ${ }^{12)}$ reported that the error rate and speed of mirror imitation was better and faster than anatomical imitation, respectively. These reports suggest the different imitation models can be classified into the following four types: 1) third person perspective mirror imitation (3PM) in which the performer and the model are positioned face-to-face with each other 
and the performer is instructed to imitate the model as if looking in a mirror; 2) third person perspective anatomical imitation (3PA) in which the performer and the model are positioned face-to-face to each other and the performer is instructed to imitate the contralateral limb movements of the model; 3) first person perspective ipsilateral imitation (1PI) in which the performer is positioned behind the model and is instructed to imitate the ipsilateral limb of the model; and 4) first person perspective contralateral imitation (1PC) in which the performer is positioned behind the model and is instructed to imitate the contralateral limb of the model. Watanabe et al. ${ }^{13)}$ examined the effect of these four imitation models (3rd person congruent or 3PM, 3rd person incongruent or 3PA, 1st person congruent or 1PI, and 1st person incongruent or 1PC) based on the measurements of reaction time and brain activity scanned by functional MRI during a finger-lifting task. They concluded that the first person views were more than adequate for facilitating imitation behavior. Our professional opinion is in agreement with Watanabe et al. that there are four valid imitation models; however, the 1 st person incongruent model (1PC) is not widely used in clinical practice because it causes confusion in young patients, and it was, therefore, excluded from the present investigation.

In previous studies comparing the differences in the efficiency of the imitation models between 3PM and 3PA, 3PM was reported to have a higher accuracy and greater speed of imitation than $3 \mathrm{PA}^{10,11,14,15)}$. Based on these previous studies, our hypothesis was that 1PI would be a more effective imitation model than 3PM and 3PA, because the information processing of 1PI would consist of egocentric views without mental rotation.

Few studies have compared the differences in the accuracy and speed of imitation among the three imitation models (1PI, 3PM and 3PA). In addition, previous studies did not assess the subjective ease of imitation of movement among the three models. Furthermore, photographs of small body parts such as the hand were used as the object of the movement imitation in previous studies, and this may not be appropriate in physical therapy since imitation of the whole body movement is often necessary. Therefore, the purpose of this study was to compare the most effective imitation model by comparing the accuracy, speed and subjective ease of imitation among 1PI, 3PM and 3PA in the imitation of a whole body movement.

\section{SUBJECTS AND METHODS}

Thirty-four right-handed healthy males aged 18 to 37 years, who gave their informed consent, participated in this study. This study was approved by the ethics committee of the School of Medicine, Shinshu University, Japan (No. 1573).

The imitation model was given by the projection of figures on a screen by commercial software (POSER7, e frontier, Inc., Japan) (Fig.1). Each figure consisted of an asymmetrical posture through the combined movement of the four extremities that were randomly presented in order to minimize the ability of the subjects to predict the sequence. Subjects stood facing the screen at a distance of $2 \mathrm{~m}$. First, the words

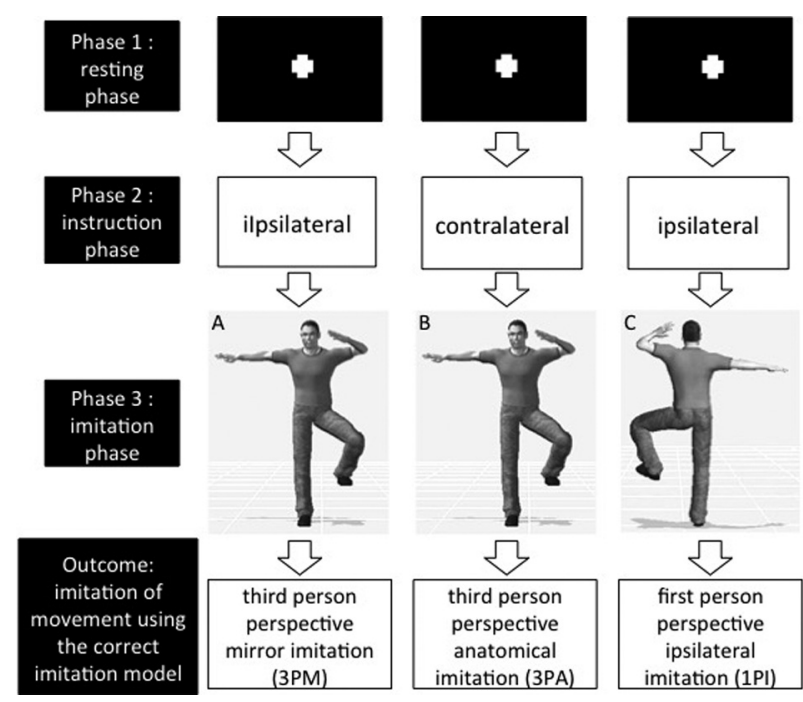

Fig. 1. Type and sequence of imitation

These figures indicate the three types of imitation and the sequence of imitation in individual imitation task. The imitation task of 3PM required ipsilateral limb movement of the model (A), as if looking in a mirror. The imitation task of 3PA required contralateral limb movement of the model (B). The imitation task of 1PI required ipsilateral limb movement behind the model (C).

"ipsilateral" or "contralateral" were displayed on the screen. Following this, one of two types of figures in which one faced towards the subject and the other faced away from the subject was displayed on the screen. Depending on whether the words displayed were "ipsilateral" or "contralateral" and the figure displayed was facing "towards" or "away" from the subjects, they would then perform the imitation of the movement pattern as accurately and as quickly as possible according to one of the three imitation models (1PI, 3PM or 3PA). For example, if the word displayed was "ipsilateral" and the figure was facing "away", then subjects had to imitate the movement according to the 1PI imitation model. If the word displayed was "ipsilateral" and the figure was facing "towards", then subjects had to imitate the movement according to the 3PM imitation model. If the word displayed was "contralateral" and the figure was facing "towards", then subjects had to imitate the movement according to 3PA imitation model.

One trial of imitation movement consisted of the following three phases (Fig. 1): 1) resting phase, looking at the blank screen displayed for 5 seconds; 2) instruction phase, looking at the words "ipsilateral" or "contralateral" displayed for 3 seconds; 3 ) imitation phase, imitating the displayed figure for 6 seconds according to the subjects' perception of the correct imitation model. All subjects were instructed to accurately and quickly imitate the movement as best as possible based on the contents of the imitation model projected on the screen in front of them. Subjects performed 1 set which consisted of 1 trial of each of the three imitation models (1PI, 3PM and 3PA). A total of 4 sets (i.e. 12 trials) were carried out, and the order of display for each of the three imitation models within each set was randomized according to the Latin square method. We described the whole 
Table 1. Comparison of the correct response ratio, reaction time and subjective easiness of 3PM, 3PA and 1PI

\begin{tabular}{|c|c|c|c|c|c|c|}
\hline & & mean \pm SD & & $3 \mathrm{PM}^{-3 \mathrm{PA}^{\mathrm{a}}}$ & $3 \mathrm{PM}-1 \mathrm{PI}^{\mathrm{a}}$ & 1PI-3PA \\
\hline & 3PM & 3PA & 1PI & & & \\
\hline Correct response ratio (\%) & $98.3 \pm 5.23$ & $90.0 \pm 17.8$ & $98.3 \pm 5.24$ & $*$ & & \\
\hline Reaction time (msec) & $0.99 \pm 0.43$ & $1.20 \pm 0.52$ & $0.90 \pm 0.27$ & $*$ & * & * \\
\hline 3-grade ordinal scale (score) & $22.3 \pm 5.59$ & $12.7 \pm 6.29$ & $25.0 \pm 7.71$ & $*$ & & $*$ \\
\hline
\end{tabular}

${ }^{a}$ Wilcoxon signed rank sum test based on the Bonferroni correction. ${ }^{*} \mathrm{p}<0.017$

3PM: third person perspective mirror imitation; 3PA: third person perspective anatomical imitation; 1PI: first person perspective ipsilateral imitation

procedure of the experiment to the subjects verbally, and subjects were allowed to practice the three types of imitation models once.

Measurement of the accuracy was done by observational assessment by one examiner viewing videotape recordings of the subjects. Accuracy was determined by counting the number of correct responses in the 4 sets. A correct response ratio (CRR) was calculated based on the ratio of the number of correct response to total trial number (i.e. 12). Assessment of the speed of imitation was done by measuring the reaction time which was determined from the start of the onset signal from a specially made external controller (ME Co. Ltd., Japan) which was used to advance the slides in the Powerpoint presentation, to the onset of the signal from an accelerometer attached to the right hand of the subject. The reaction time was calculated as the duration between the onset of the trigger signal in the external controller and the onset of the movement artifact in the accelerometer, by using bio-information analyzing software (BIMUTAS-E, version2.0, Kissei-comtec Ltd., Japan). Evaluation of the subjective ease of imitation was done based on a questionnaire that ranks the ease of imitation movement among the three conditions immediately after the experiment. Rankings of subjective ease of imitation were classified according to the following three grade ordinal scale: Grade 1, easiest to imitate; Grade 2, easier to imitate; and Grade 3, easy to imitate. Analysis of the 3-grade ordinal scale was done by calculation of the mean value of a weighting score as follows: Grade 1 was given 30 points, Grade 2 was given 20 points, and Grade 3 was given 10 points for the statistical analysis.

Statistical analysis of CRR, reaction time and the 3-grade ordinal scale was carried out using the Wilcoxon signed rank sum test with Bonferroni correction. Results are expressed as means and standard deviation (SD). Statistical significance was chosen as 0.017 (after Bonferroni correction). All statistical analyses were performed using IBM SPSS version 22 (IBM Corporation, New York, USA).

\section{RESULTS}

Table 1 shows the comparison among 1PI, 3PM and 3PA of CRR, reaction time and the 3-grade ordinal scale. For CRR, the results show that 3PM had significantly higher scores than 3PA $(p=0.008)$. For reaction time, the results show that 1PI was performed significantly quicker than 3PM $(p=0.01)$ and 3PA $(p=0.001)$, and 3PM was performed significantly quicker than $3 \mathrm{PA}(\mathrm{p}=0.001)$. For the 3 -grade or- dinal scale subjective ease of imitation, the results show that 1PI was significantly easier to imitate than $3 \mathrm{PA}(\mathrm{p}=0.001)$, and $3 \mathrm{PM}$ was significantly easier than $3 \mathrm{PA}(\mathrm{p}=0.001)$.

\section{DISCUSSION}

The purpose of this study was to find the most effective imitation model (1PI, 3PM and 3PA) by comparing CRR as an index of accuracy, reaction time as the speed of imitation, and the 3-grade ordinal scale as the subjective ease of imitation. The results show that $3 \mathrm{PM}$ was the most accurate, but 1PI was the quickest and also the easiest to imitate.

Regarding the accuracy of imitation, Philip et al. ${ }^{8)}$ reported that mirror imitation is the most common paradigm, since mirror imitation is an innate natural reaction, and easier to remember than anatomical imitation. Chiavarino et al. ${ }^{12)}$ reported that when they examined accuracy of imitation using mirror-image and anatomical imitation in a task of manipulating objects, mirror-image imitation was significantly easier than anatomical imitation $(82.4 \%$ correct versus $56.7 \%$ correct). The results of these studies suggest that the 3PM and 1PI imitation models facilitate accurate imitation of movement because mental rotation is not required.

Regarding the speed of the imitation of movement, Conson et al. ${ }^{16)}$ examined the reaction times of subjects with Asperger syndrome and healthy controls who performed mental rotation. The subjects were asked to decide whether a rotated hand image was left or right (i.e. hand laterality task), as well as mental rotation of alphabet letters. Their results show that the speed of judgment of hand laterality and mental rotation of alphabet letters was greater when the rotation angle of the displayed objects were minimal. Since the results of the reaction time in the present study were similar to those of Conson et al. ${ }^{16)}$, it is our opinion that the imitation model without mental rotation has a quicker reaction time than the imitation model with mental rotation.

Regarding the subjective ease of imitation, Krause et al. ${ }^{17)}$ reported that lower disparities between the model's and the observer's egocentric perspectives would result in better reproductions of movement kinematics by the imitator. Imitation with less angle of mental rotation may require less cognitive processing; therefore, imitating the movement patterns by observation could be perceived as easier to execute $^{13,18,19)}$. The results of the present study also suggest that imitation models without mental rotation, such as 3PM and 1PI, are easier to imitate than an imitation model with mental rotation, such as 3PA.

In general, our results show that imitation models without 
mental rotation, such as $3 \mathrm{PM}$ and $1 \mathrm{PI}$, are more accurate, faster and easier than those with mental rotation, such as 3PA. However, which of 3PM and 1PI is the better imitation model? Philip et al. ${ }^{8)}$ reported that 1PPI was more accurate and faster than 3PPI. Their results supported their hypothesis that the more visuospatial similarity between the imitator and the model to imitate, the easier the task, in line with the "Like-Me" mechanism of imitation ${ }^{20-22)}$ (which is the projection of how infants use self-experience as leverage for understanding the behavior of others who act "like me"20)). Moreover, Philip et al. ${ }^{8}$ ) suggested that the first person perspective recruits the motor system more extensively than the third person perspective, regardless of whether the movements are produced or not, and this is fully compatible with ideomotor theories ${ }^{23)}$.

Collectively, the results of these previous studies suggest that 1PI is the best imitation model compared to the others in this study. However, it is not possible to definitively make this claim since there were discrepancies in the results, such as 3PM showing high accuracy, 1PI showing faster reaction time and a higher score of subjective ease in imitation.

There were several limitations to our study. First, it was possible that subjects did not fully understand the contents of the imitation task because of inadequate practice, since the subjects were only allowed one practice for each imitation model. Second, the design of the study was a descriptive cross-sectional study that did not allow for a period of intervention using each of the three different imitation models. Therefore, further studies may be necessary to clarify the effects of imitation models based on a longitudinal clinical study of actual patients.

\section{REFERENCES}

1) Kim J, Lee B, Lee HS, et al.: Differences in brain waves of normal persons and stroke patients during action observation and motor imagery. J Phys Ther Sci, 2014, 26: 215-218. [Medline] [CrossRef]

2) Mataric MJ: Motor learning by Imitation. AAAI Tech Rep, 1994, FS-9403: $132-136$.

3) Ohno K, Higashi T, Sugawara K, et al.: Excitability changes in the human primary motor cortex during observation with motor imagery of chopstick use. J Phys Ther Sci, 2011, 23: 703-706. [CrossRef]

4) Kim JS, Kim K: Clinical feasibility of action observation based on mirror neuron system on walking performance in post stroke patients. J Phys Ther Sci, 2012, 24: 597-599. [CrossRef]

5) Al-Abood SA, Davids KF, Bennett SJ: Specificity of task constraints and effects of visual demonstrations and verbal instructions in directing learners' search during skill acquisition. J Mot Behav, 2001, 33: 295-305. [Medline] [CrossRef]

6) Kim JH, Chung EJ, Lee BH: A study of analysis of the brain wave with respected to action observation and motor imagery: a pilot randomized controlled trial. J Phys Ther Sci, 2013, 25: 779-782. [Medline] [CrossRef]

7) Park EC, Hwangbo G: The effects of action observation gait training on the static balance and walking ability of stroke patients. J Phys Ther Sci, 2015, 27: 341-344. [Medline] [CrossRef]

8) Jackson PL, Meltzoff AN, Decety J: Neural circuits involved in imitation and perspective-taking. Neuroimage, 2006, 31: 429-439. [Medline] [CrossRef]

9) Shepard RN, Metzler J: Mental rotation of three-dimensional objects. Science, 1971, 171: 701-703. [Medline] [CrossRef]

10) Franz EA, Ford S, Werner S: Brain and cognitive processes of imitation in bimanual situations: Making inferences about mirror neuron systems. Brain Res, 2007, 1145: 138-149. [Medline] [CrossRef]

11) Koski L, Iacoboni M, Dubeau MC, et al.: Modulation of cortical activity during different imitative behaviors. J Neurophysiol, 2003, 89: 460-471. [Medline] [CrossRef]

12) Chiavarino C, Apperly IA, Humphreys GW: Exploring the functional and anatomical bases of mirror-image and anatomical imitation: the role of the frontal lobes. Neuropsychologia, 2007, 45: 784-795. [Medline] [CrossRef]

13) Watanabe R, Higuchi T, Kikuchi Y: Imitation behavior is sensitive to visual perspective of the model: an fMRI study. Exp Brain Res, 2013, 228: 161-171. [Medline] [CrossRef]

14) Belopolsky AV, Olivers CN, Theeuwes J: To point a finger: attentional and motor consequences of observing pointing movements. Acta Psychol (Amst), 2008, 128: 56-62. [Medline] [CrossRef]

15) Richter S, Brenner E, Karnath HO: Movement orientation is related to mental rotation in childhood. Dev Neuropsychol, 2009, 34: 284-295. [Medline] [CrossRef]

16) Conson M, Mazzarella E, Frolli A, et al.: Motor imagery in Asperger syndrome: testing action simulation by the hand laterality task. PLoS ONE, 2013, 8: e70734. [Medline] [CrossRef]

17) Krause D, Kobow $S$ : Effects of model orientation on the visuomotor imitation of arm movements: the role of mental rotation. Hum Mov Sci, 2013, 32: 314-327. [Medline] [CrossRef]

18) Neely KA, Heath M: Visuomotor mental rotation: reaction time is determined by the complexity of the sensorimotor transformations mediating the response. Brain Res, 2010, 1366: 129-140. [Medline] [CrossRef]

19) Neely KA, Heath M: Visuomotor mental rotation: the reaction time advantage for anti-pointing is not influenced by perceptual experience with the cardinal axes. Exp Brain Res, 2010, 201: 593-598. [Medline] [CrossRef]

20) Meltzoff AN: 'Like me': a foundation for social cognition. Dev Sci, 2007, 10: 126-134. [Medline] [CrossRef]

21) Meltzoff AN: Imitation and other minds: the "Like Me" hypothesis. In: Hurley S, Chater N (eds.): Perspectives on Imitation: From Cognitive Neuroscience to Social Science. Cambridge: MIT Press, 2005, pp 55-77.

22) Meltzoff AN, Decety J: What imitation tells us about social cognition: a rapprochement between developmental psychology and cognitive neuroscience. Philos Trans R Soc Lond B Biol Sci, 2003, 358: 491-500. [Medline] [CrossRef]

23) Greenwald AG: Sensory feedback mechanisms in performance control: with special reference to the ideo-motor mechanism. Psychol Rev, 1970, 77: 73-99. [Medline] [CrossRef] 